\title{
CÂNCER PEDIÁTRICO: PERFIL EPIDEMIOLÓGICO DOS PACIENTES ATENDIDOS NO SERVIÇO DE ONCOLOGIA PEDIÁTRICA DO HOSPITAL DE CLÍNICAS DA UFPR
} CHILDHOOD CANCER: EPIDEMIOLOGICAL PROFILE OF PATIENTS REFERRED TO THE HOSPITAL DE CLIINICAS OF UFPR PEDIATRIC ONCOLOGY UNIT. DOI: $10.5380 /$ rmu.v1i4.40690

Tania Cristine Hadas', Adriane Elizabeth Gamarra Gaete', Mara Albonei Dudeque Pianovski.

\section{RESUMO}

Objetivo: Avaliar epidemiologicamente os dados das crianças atendidas no Hospital de Clínicas da Universidade Federal do Paraná, diagnosticadas com câncer em um período de 10 anos. Métodos: Análise dos dados de 424 pacientes, de 0 a 14 anos, diagnosticados no período de 2001 a 2010. Foram excluídos da análise de sobrevida os pacientes com diagnóstico realizado após $31 / 12 / 2009$, aqueles com carcinoma de suprarrenal e os que realizaram tratamento prévio em outros serviços. Resultados: Do total, 211 (49,8\%) eram do sexo masculino e $213(50,2 \%)$ do sexo feminino. Em relação à distribuição dos pacientes de acordo com a faixa etária, $30(7,1 \%)$ eram menores de 1 ano, $179(42,2 \%)$ de 1 a 4 anos, $137(32,3 \%)$ de 5 a 9 anos e $78(18,4 \%)$ de 10 a 14 anos. Em ordem de frequência, ficaram em primeiro lugar as leucemias (46,0\%), seguidas pelos linfomas (13,0\%), retinoblastomas $(7,8 \%)$ e tumores de sistema nervoso central (SNC) (6,0\%). Os tumores sólidos em geral corresponderam a 174 casos $(41,0 \%)$. Foram incluídos 316 casos na análise de sobrevida, dentre os quais $220(69,6 \%)$ estavam vivos ao final de 5 anos de seguimento, 92 (29,1\%) foram a óbito pela neoplasia ou por causas a ela relacionadas e 4 (1,3\%) por outras causas. A sobrevida global em 5 anos foi de $74,1 \%$ para as leucemias, $78,2 \%$ para os linfomas e $67,9 \%$ para os tumores sólidos. Conclusão: A análise de sobrevida das leucemias está em concordância com os dados de estudos de países desenvolvidos.

Palavras-chave: Registro de câncer. Câncer infantil. Sobrevida. Neoplasias. Pediatria.

\begin{abstract}
Background: Epidemiologically evaluate the data from children referred to the Hospital de Clinicas of Universidade Federal do Paraná (HC-UFPR) with cancer diagnosis in a period of ten years. Methods: The data of 424 patients, 0-14 years old, with natural origin of the country or abroad, diagnosed on the period of 2001 to 2010 were assessed. Patients with diagnosis made after 2009 Dec 31st, or with adrenal carcinoma or who underwent pretreatment in other services were excluded from the survival analysis. Results: Of the total, 211 (49.8\%) were male and 213 were female (50.2\%). Regarding the distribution of patients according to age group, $30(7.1 \%)$ were younger than 1 year, 179 (42.2\%) 1-4 years old, $137(32.3 \%)$ 5-9 years and $78(18.4 \%)$ 10-14 years. In order of frequency, first were leukemias (46.0\%), followed by lymphomas (13.0\%), retinoblastomas $(7.8 \%)$ and tumors of the central nervous system (6.0\%). A total of 316 cases were included in the survival analysis, among which $220(69.6 \%)$ were alive at 5 years follow-up, $92(29.1 \%)$ died by the neoplasm or by causes related to it and $4(1.3 \%)$ from other causes. The overall 5 -year survival was $74.1 \%$ for leukemias, $\mathbf{7 8 . 2 \%}$ for lymphomas and 67.9 \% for solid tumors. Conclusions: Survival analysis of leukemia is in agreement with data from studies conducted in developed countries.
\end{abstract}

Key Words: Cancer registry, Childhood cancer, Survival, Neoplasms, Pediatrics.

\section{1 - Universidade Federal do Paraná}

Contato do Autor / Mail to:

Ambulatório Menino Jesus de Praga - albonei@yahoo.com.br

Rua General Carneiro, 6, Alto da Glória, 80060-900 - Curitiba - PR 


\section{REVISTA MÉDICA DA UFPR}

\section{INTRODUÇÃO}

O câncer pediátrico é uma grande preocupação de saúde pública constituindo-se na segunda causa de mortalidade proporcional na faixa etária de 1 a 19 anos, seguindo-se aos óbitos por acidentes e violência ${ }^{1,2}$. Representa cerca de $2 \%$ de todas as neoplasias malignas ${ }^{3}$. No Brasil, estima-se que há cerca de nove mil casos novos por ano de câncer em crianças e adolescentes ${ }^{1}$.

É estudado e classificado de maneira distinta do câncer do adulto, baseando-se na morfologia e não no sítio de origem. Mundialmente, utiliza-se a Classificação Internacional do Câncer na Infância (International Classification of Childhood Cancer - ICCC), criada em 1996, hoje em sua terceira edição (2005), de acordo com as novas classificações morfológicas propostas na CID-O$3(2000)^{4}$.

Há diferenças a se considerar entre o câncer na faixa pediátrica e no adulto. Na criança, afeta o sistema hematopoético e os tecidos de sustentação, enquanto no adulto há preferência pelo epitélio que recobre os diferentes órgãos ${ }^{5}$. Não se associa com fatores de risco comportamentais e ambientais, como se verifica com as neoplasias do adulto (tabagismo, etilismo, exposição ao sol, entre outros). O câncer pediátrico costuma também ser mais invasivo, apresenta menor período de latência e maior velocidade de crescimento. Apesar dessas características, geralmente responde bem ao tratamento e é considerado de bom prognóstico ${ }^{6}$.

Os estudos epidemiológicos servem de base para avaliar a qualidade dos serviços e criar novas estratégias em programas de controle de câncer em saúde pública - a incidência, a prevalência, mortalidade e taxas de sobrevida são importantes indicadores de qualidade do sistema de saúde ${ }^{7}$. Em relação ao câncer pediátrico, tais estudos são menos frequentes se comparados aos do adulto. Essas análises visam planejamento e melhora no atendimento, diagnóstico e tratamento dos pacientes $^{8}$.

\section{MÉTODOS}

Estudo ambispectivo, realizado no ambulatório do serviço de oncologia pediátrica do Hospital de Clínicas da Universidade Federal do Paraná (HC-UFPR), mediante construção de um banco de dados a partir dos prontuários dos pacientes desse serviço.

O projeto foi aprovado pelo Comitê de Ética em Pesquisa em Seres Humanos do HCUFPR com dispensa do Termo de Consentimento Livre e Esclarecido porque o estudo não requereu intervenção no paciente ou coleta de material biológico, além da possibilidade de constrangimentos aos familiares caso o paciente tenha ido a óbito.

Foram incluídos no estudo os dados de 424 pacientes, com idade até 14 anos, com procedência natural do país ou exterior, que procuraram o referido serviço para diagnóstico e tratamento do câncer; este foi confirmado por métodos diagnósticos de imagem, citológicos ou histopatológicos, no período de 01/01/2001 a 31/12/2010. Não foram incluídos no estudo dados dos 9 pacientes portadores de neoplasias benignas atendidos no período. Os pacientes com diagnóstico realizado após 31/12/2009 foram excluídos da análise de sobrevida, assim como aqueles que realizaram tratamento prévio em outros serviços. Os pacientes com diagnóstico de carcinoma de suprarrenal também foram excluídos da análise de sobrevida porque no serviço de oncologia pediátrica do HC-UFPR são tratados apenas os pacientes com estadio III ou IV, sendo os pacientes com estadio I ou II acompanhados no serviço de endocrinopediatria por não necessitarem de quimioterapia. A sua inclusão causaria um viés de análise.

A coleta de dados foi realizada nos meses de junho de 2013 a abril de 2014 a partir de prontuários alocados no ambulatório de oncologia pediátrica e arquivo do HC-UFPR. As variáveis utilizadas foram sexo, idade, local de nascimento, grupo pediátrico segundo ICCC, data do primeiro diagnóstico, situação clínica atual, data da última consulta, e caso tenha ocorrido, a data do óbito. A classificação do ICCC divide o câncer pediátrico em 12 grupos principais, baseando-se na morfologia e sítio primário do tumor ${ }^{4}$.

Para análise de sobrevida global, dos 316 pacientes incluídos, foi utilizado o tempo entre a data do diagnóstico e a data da última consulta ou a data do óbito em decorrência do câncer ou por causas não relacionadas para os indivíduos censurados.

O banco de dados foi construído com o editor de planilhas Microsoft Office Excel. Para a análise do tempo de sobrevida utilizou-se o método de Kaplan-Meier. Para verificar a interferência da idade no prognóstico dos pacientes com leucemia linfoide aguda (LLA) foi 
aplicada análise com Regressão-Cox ${ }^{10}$. Utilizouse o teste $Z$ para verificar as diferenças de proporções relativas ao sexo, de acordo com o grupo pediátrico. O programa computacional utilizado para o processamento de todos esses dados foi $R$ versão 3.0.1.

\section{RESULTADOS}

Dos 424 pacientes incluídos no estudo, 211 (49,8\%) eram do sexo masculino e 213 $(50,2 \%)$ do sexo feminino. Em relação à distribuição dos pacientes de acordo com a faixa etária, 30 (7\%) eram menores de 1 ano, 179 (42\%) tinham de 1 a 4 anos, 137 (32\%), de 5 a 9 anos e 78 (18\%), de 10 a 14 anos.

De acordo com o local de nascimento, 394 (92,9\%) eram da Região Sul, sendo 378 (89,2\%) do estado do Paraná, 14 (3,3\%) de Santa Catarina e $2(0,5 \%)$ do Rio Grande do Sul. Em relação às demais regiões do país, $7(1,6 \%)$ eram do Norte, 2 (0,5\%) do Nordeste, 9 (2,1\%) do Sudeste e $9(2,1 \%)$ do Centro-Oeste. Houve $3(0,7 \%)$ casos do exterior, sendo 2 casos do Paraguai e 1 da Itália.

As neoplasias mais frequentes foram as leucemias com 195 casos (46,0\%), seguidas pelos linfomas com 55 (13\%), retinoblastomas com $33(7,8 \%)$ e tumores de sistema nervoso central (SNC) com 25 (6,0\%). Os tumores sólidos em geral corresponderam a 174 casos (41,0\%) (TABELA 1).

No grupo dos linfomas, houve predomínio do sexo masculino, com 36

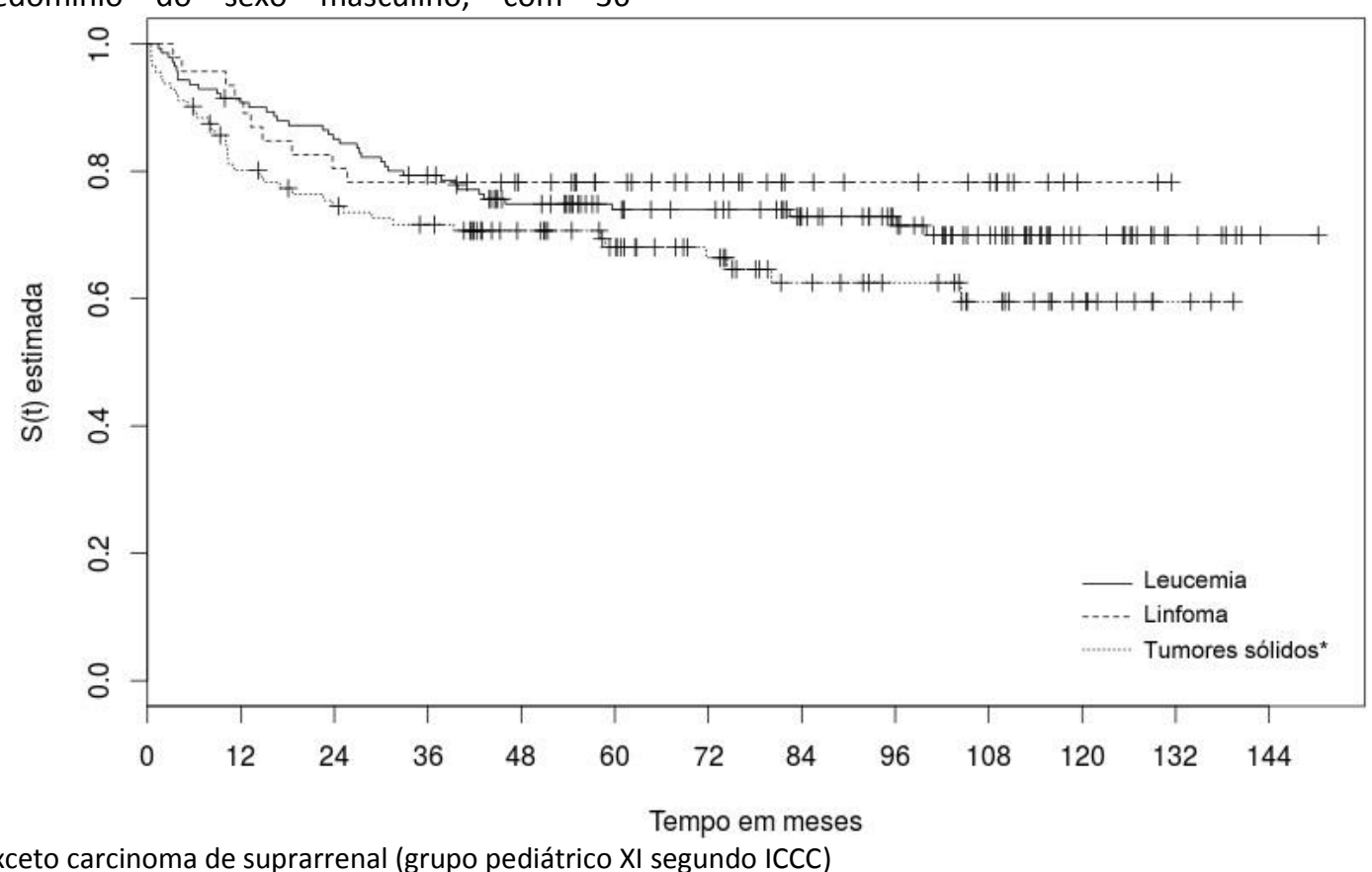

pacientes (65,5\%), diferença essa estatisticamente significativa $(p<0,05)$.

Entre os 195 casos de leucemias, identificaram-se 133 (68,2\%) de LLA, 48 (24,6\%) de leucemia não linfoide aguda (LNLA) e 13 $(6,7 \%)$ de leucemia mieloide crônica (LMC). Houve um caso de leucemia do tipo bifenotípica. Quanto aos linfomas, 30 casos $(54,6 \%)$ foram classificados como não-Hodgkin (LNH) e $25(45,4 \%)$ como linfoma de Hodgkin (LH).

$\mathrm{Na}$ análise de sobrevida foram incluídos 316 casos, dentre os quais 220 $(69,6 \%)$ estavam vivos ao final de 5 anos de seguimento, 92 (29,1\%) foram a óbito pela neoplasia ou por causas a ela relacionadas e 4 $(1,3 \%)$ por outras causas diversas. A sobrevida global em 5 anos foi de $74,1 \%$ para as leucemias, $78,2 \%$ para os linfomas e $67,9 \%$ para os tumores sólidos (FIGURA 1).

Em relação às leucemias, a sobrevida global em 5 anos foi de 81,8\% para LLA, 53,2\% para LNLA e 67,5\% para LMC (FIGURA 2). Para pacientes com diagnóstico de linfoma, a sobrevida foi de $71,0 \%$ para os $\mathrm{LNH}$ e $83,9 \%$ para os LH (FIGURA 3).

Ao testarmos a influência da idade ao diagnóstico em relação ao prognóstico da LLA não foi encontrada diferença estatística significativa.
Exceto carcinoma de suprarrenal (grupo pediátrico XI segundo ICCC) 


\section{REVISTA MÉDICA DA UFPR}

Figura 1 - Sobrevida global em 5 anos dos pacientes com diagnóstico realizado de 2001 a 2009, de acordo com os tipos de câncer.

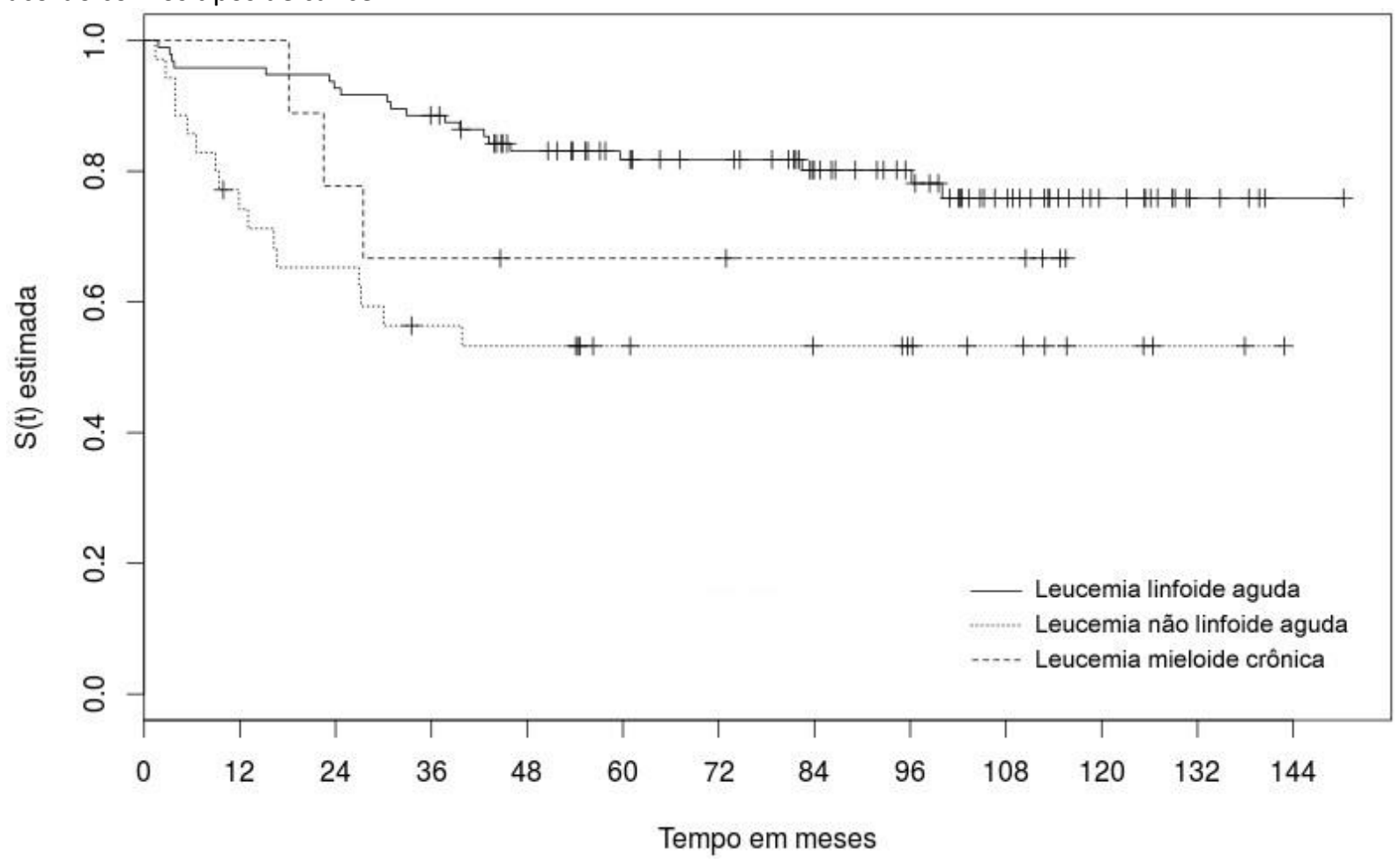

Figura 2 - Sobrevida global em 5 anos dos pacientes com diagnóstico realizado de 2001 a 2009, de acordo com o tipo de leucemia.

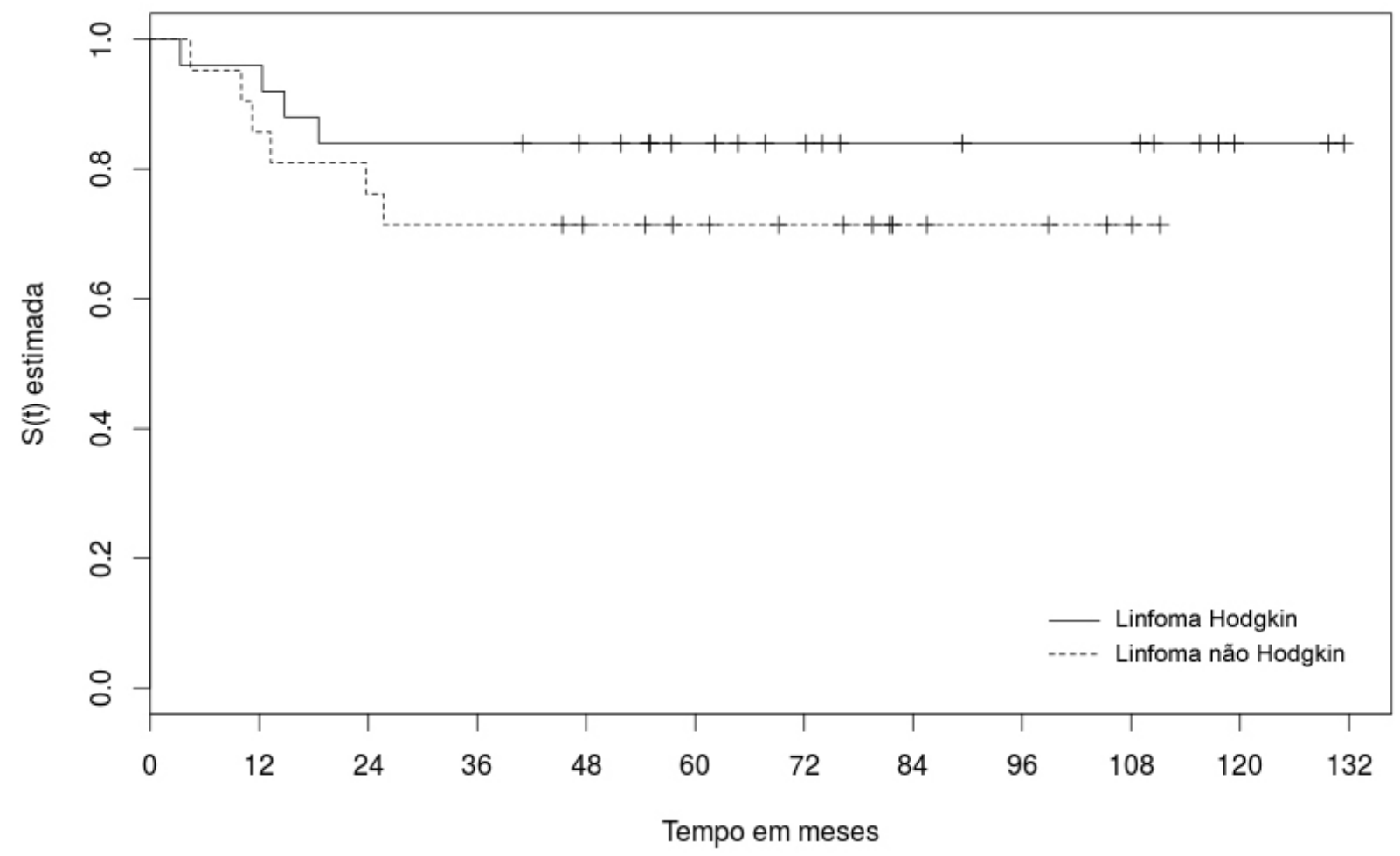

Figura 3 - Sobrevida global em 5 anos dos pacientes com diagnóstico realizado de 2001 a 2009, de acordo com o tipo de linfoma 
Tabela 1 - Distribuição das neoplasias segundo o grupo diagnóstico (iccc) e o sexo

\begin{tabular}{|c|c|c|c|c|c|c|}
\hline \multirow{3}{*}{ GRUPO AO DIAGNÓSTICO SEGUNDO ICCC* } & \multicolumn{4}{|c|}{ SEXO } & \multirow{2}{*}{\multicolumn{2}{|c|}{$\begin{array}{l}\text { TOTAL POR } \\
\text { GRUPO }\end{array}$}} \\
\hline & \multicolumn{2}{|c|}{ MASCULINO } & \multicolumn{2}{|c|}{ FEMININO } & & \\
\hline & $\mathrm{n}$ & $\%$ & $\mathrm{n}$ & $\%$ & $\mathrm{n}$ & $\%$ \\
\hline $\begin{array}{l}\text { I. Leucemias, doenças mieloproliferativas e doenças } \\
\text { mielodisplásicas }\end{array}$ & 97 & 49,7 & 98 & 50,3 & 195 & 46,0 \\
\hline II. Linfomas e neoplasias reticuloendoteliais & 36 & 65,5 & 19 & 34,5 & 55 & 13,0 \\
\hline $\begin{array}{l}\text { III. SNC e miscelânia de neoplasias intracranianas e } \\
\text { intraespinhais }\end{array}$ & 15 & 60 & 10 & 40 & 25 & 5,9 \\
\hline $\begin{array}{l}\text { IV. Neuroblastoma e outros tumores de células } \\
\text { nervosas periféricas }\end{array}$ & 10 & 52,6 & 9 & 47,4 & 19 & 4,5 \\
\hline V. Retinoblastoma & 13 & 39,4 & 20 & 60,6 & 33 & 7,8 \\
\hline VI. Tumores renais & 9 & 45 & 11 & 55 & 20 & 4,7 \\
\hline VII. Tumores Hepáticos & 0 & 0 & 4 & 100 & 4 & 0,9 \\
\hline VIII. Tumores ósseos malignos & 7 & 53,8 & 6 & 46,2 & 13 & 3,1 \\
\hline IX. Tecidos moles e outros sarcomas extra-ósseos & 4 & 33,3 & 8 & 66,7 & 12 & 2,8 \\
\hline $\begin{array}{l}\text { X. Tumores de células germinativas, tumores } \\
\text { trofoblásticos, e neoplasias gonadais }\end{array}$ & 3 & 37,5 & 5 & 62,5 & 8 & 1,9 \\
\hline $\begin{array}{l}\text { XI. Outros neoplasmas malignos epiteliais e outros } \\
\text { melanomas malignos }\end{array}$ & 7 & 30,4 & 16 & 69,6 & 23 & 5,4 \\
\hline XII. Outras neoplasias malignas e não especificadas & 10 & 58,8 & 7 & 41,2 & 17 & 4,0 \\
\hline TOTAL & 211 & 100 & 213 & 100 & 424 & 100 \\
\hline
\end{tabular}

*International Classification of Childhood Cancer (ICCC) 


\section{DISCUSSÃO}

As leucemias são o tipo de câncer
pediátrico mais comum no mundo. Correspondem a $34,1 \%$ de todas as neoplasias infantis até os 15 anos de idade, sendo seguidas pelos tumores do SNC $(22,6 \%)$ e pelos linfomas $(11,5 \%)^{2}$. Um estudo nacional que reuniu 14 registros de câncer de base populacional (RCBP) mostrou que Goiânia, Manaus e Curitiba eram as 3 capitais com maiores taxas de incidência para leucemia ${ }^{7}$. No presente estudo também foi a neoplasia mais frequente. Dependendo do local e da população analisada, as incidências de linfomas e tumores do SNC podem variar, alternando posições como segundo e terceiro tipo de câncer infantil mais frequente. No Brasil, os linfomas aparecem como a segunda neoplasia mais incidente na infância ${ }^{7}$.

$\mathrm{Na}$ população analisada, os linfomas foram a segunda neoplasia mais frequente, seguidos do retinoblastoma e tumores do SNC. O retinoblastoma é o tumor sólido infantil mais comum em alguns países subdesenvolvidos e em desenvolvimento e pode ter inclusive uma incidência maior do que a de neuroblastomas, que são sempre mais comuns em países desenvolvidos ${ }^{11}$. Contudo, o número de casos de tumores do SNC pode não corresponder à realidade desta população geográfica regional, uma vez que existem outros serviços em Curitiba que também são referência para o diagnóstico e tratamento de tumores do SNC. Em serviços analisados nos estados de Santa Catarina e Porto Alegre, os tumores de SNC apareceram em segundo na ordem de frequência, seguido dos linfomas ${ }^{8,12}$. Já no estudo nacional dos RCBP, eles estão em terceiro lugar? ${ }^{7}$.

Em trabalhos realizados em serviços hospitalares dos estados de Santa Catarina e Rio Grande do Sul, encontraram-se 36,6\% e $26,9 \%$, respectivamente, de incidência para leucemia ${ }^{8,12}$. No serviço de oncologia pediátrica do HC-UFPR, 46\% corresponderam a esse grupo. Deve-se considerar o fato de o HC-UFPR ser um dos principais serviços responsáveis pelo atendimento da população oncológica pediátrica da capital e do estado do Paraná. É uma instituição de referência no diagnóstico de leucemias e na área de transplante de medula óssea, tendo sido o pioneiro na realização deste procedimento na América Latina em $1979^{13}$.

Em geral, as leucemias são mais frequentes de 1 a 9 anos. Para o subgrupo da LLA, há um pico entre 2 e 3 anos $^{3}$, o qual ocorreu em $66 \%$ dos casos analisados. 0 linfoma é praticamente inexistente em menores de um ano, raro em crianças de 1 a 4 anos, tendo maiores frequências nas faixas etárias seguintes ${ }^{2}$. No presente estudo, não houve nenhum caso em menores de 1 ano, $12,7 \%$ apresentaram-se de 1 a 4 anos, $49,1 \%$ de 5 a 9 anos e $38,2 \%$ de 10 a 14 anos. 0 diagnóstico dos retinoblastomas, em $2 / 3$ dos casos, é feito em crianças menores de 2 anos $^{3}$. Nesse estudo, cerca de $57,5 \%$ dos casos foram diagnosticados nessa faixa de idade. Aproximadamente $1 / 4$ desses tumores são bilaterais ao diagnóstico ${ }^{14}$ e, da mesma forma que na literatura, foram encontrados $24,2 \%$ de retinoblastomas bilaterais.

Vários estudos relatam maior incidência de câncer em geral no sexo masculino ${ }^{2,3,15}$. Na população estudada, não houve diferença estatística significativa em relação a essa variável. Para o grupo dos linfomas, como é descrito pela literatura, houve uma incidência maior no sexo masculino (65,5\%).

Registros de base populacional de 19 países europeus apresentam sobrevida em 5 anos que varia de $70 \%$ a $78 \%$ para pacientes com leucemias em geral ${ }^{16}$. No serviço avaliado, encontrou-se $74,1 \%$.

Os registros do Surveillance, Epidemiology, and End Results Program (SEER) relatam sobrevida em 5 anos de 86,1\% para LLA $^{17}$ e o Automated Childhood Cancer Information System (ACCIS), 82\% ${ }^{18}$. Nesse serviço a sobrevida em LLA foi de $81,8 \%$. Com relação a LNLA, o ACCIS encontrou uma sobrevida de $52 \%{ }^{18}$ e o serviço em questão $53,2 \%$.

A sobrevida em 5 anos para pacientes com linfoma, segundo o SEER, foi de $90 \%$, com $95,9 \%$ para $\mathrm{LH}$ e $83,8 \%$ para $\mathrm{LNH}^{17}$. $\mathrm{Na}$ população analisada pelo ACCIS, encontrou-se $85 \%$ para linfomas em geral, sendo $93 \%$ para $\mathrm{LH}$ e $79 \%$ para $\mathrm{LNH}^{18}$. No presente estudo, o grupo de pacientes com linfoma apresentou sobrevida de $78,2 \%$, com $71,0 \%$ para $\mathrm{LNH}$ e $83,9 \%$ para LH. Sabe-se que para os LH o estadiamento ao diagnóstico é uma das variáveis que influenciam o prognóstico da doença ${ }^{19}$. A mais importante variável prognóstica do LNH é a carga tumoral que é avaliada pelo estadiamento e o nível da lactato 
desidrogenase sérica ${ }^{20}$. Neste estudo, 63,0\% dos casos de linfoma analisados para sobrevida tiveram ao diagnóstico estadiamento III ou IV, o que poderia explicar a diferença encontrada. Outra variável que poderia estar relacionada com esses resultados é o comportamento biológico de cada tipo de linfoma, o qual não foi analisado no presente trabalho.

A sobrevida de 67,9\% encontrada nesse serviço para pacientes com diagnóstico de tumores sólidos indica um bom resultado. Entretanto, por ser uma amostra pequena por grupo de neoplasia, não foi possível realizar uma análise minuciosa de outras variáveis. Destaca-se que o HC-UFPR é também referência no tratamento de tumores adrenocorticais, uma vez que as maiores incidências mundiais estão nas regiões Sul e Sudeste do Brasil, podendo chegar a 4,2 casos por milhão ao ano em crianças até 15 anos de idade, enquanto mundialmente essa incidência é de 0,3 por milhão ${ }^{21,22}$.

Análises dessa mesma natureza são escassas no país. Um estudo realizado em São Paulo, no período de 1993 a 1998, relatou probabilidade de sobrevida para crianças com leucemia de apenas $41 \%^{23}$. Esse valor pode estar relacionado com o encaminhamento tardio para os centros de referência ou ainda com a realização de diagnóstico e tratamento feitos em centros não especializados no atendimento de oncologia pediátrica. Em países subdesenvolvidos e em desenvolvimento, a sobrevida só está começando a alcançar os valores de países desenvolvidos nestes últimos anos ${ }^{24}$. Esse resultado depende do diagnóstico precoce e do tratamento em centros adequadamente equipados para tais fins, sob a coordenação de recursos humanos especializados.

Os avanços nas áreas diagnósticas, terapêuticas e de suporte para as complicações inerentes ao tratamento fez com que os pacientes formassem uma nova população específica, a dos sobreviventes do câncer infantil, sujeita às complicações tardias das modalidades terapêuticas a que foram submetidas $^{25}$, como alterações estéticas leves, disfunções orgânicas e cognitivas, além de neoplasias secundárias ${ }^{26}$. É um grupo de pacientes que tende a crescer e, portanto, os médicos que provirão os diversos cuidados em saúde na vida adulta deverão estar atentos para as possíveis doenças e complicações ainda em fases subclínicas ${ }^{25}$.

\section{CONCLUSÃO}

O serviço de oncologia pediátrica do HC-UFPR é um centro de referência no diagnóstico e tratamento das neoplasias na infância. Apesar de ter as dificuldades esperadas por se tratar de um serviço público e sediado num país em desenvolvimento relativas ao acesso da população, possibilidade da família oferecer os cuidados que a criança com neoplasia necessita e à prontidão e rapidez de materiais, recursos e tecnologias - o resultado foi equiparável ao de países desenvolvidos. Para os pacientes do serviço de oncologia pediátrica do HC-UFPR, as dificuldades socioeconômicas são atenuadas pelo trabalho de voluntariado da Associação Paranaense de Apoio à Criança com Neoplasia (APACN), que fornece hospedagem adequada para as famílias de outras cidades, transporte e atendimento multiprofissional, além da estrutura de ambulatório adequada para o atendimento clínico.

\section{REFERÊNCIAS BIBLIOGRÁFICAS}

1. Instituto Nacional do Câncer. Particularidades do Câncer Infantil [Internet]. Rio de Janeiro: INCA; [citado em 2014 Jan 30]. Disponível em:

http://www.inca.gov.br/conteudo vie w.asp?id=343.

2. Kaatsch P. Epidemiology of childhood cancer. Cancer Treat Rev. 2010 Jun; 36(4):277-85.

3. Ries LAG, Smith MA, Gurney JG, Linet $M$, Tamra T, Young JL, Bunin GR (et al). Cancer Incidence and Survival among Children and Adolescents: United States SEER Program 1975-1995. Bethesda: National Cancer Institute, SEER Program; 1999.

4. Steliarova-Foucher E, Stiller C, Lacour B, Kaatsch P. International Classification of Childhood Cancer, Third Edition. Bull Am Cancer Soc. 2005 Abr 01; 103(7): 1457-67.

5. Bustos, QJ. Tumores cancerosos más frecuentes en pediatría: del oncólogo al pediatra. Pediatr. Día. 1998; 14(5): 251-8. 
6. Instituto Nacional de Câncer. Câncer no Brasil: dados dos registros de base populacional. Rio de Janeiro, 2010, INCA. p. 470.

7. de Camargo B. et al. Cancer incidence among children and adolescents in Brazil: first report of 14 populationbased cancer registries. Int. J. Cancer. 2010; 126 (3):715-20.

8. Silva DB, Pires MMS, Nassar, SM. Câncer pediátrico: análise de um registro hospitalar. J Pediatr. 2002 Set/Out;78(5):409-14.

9. Clark TG, Bradbun MJ, Love SB, Altman DG. Survival Analysis Part I: Basic concepts and first analyses. $\mathrm{Br} J$ Cancer. 2003 Jul;89(2):232-8.

10. Clark TG, Bradbun MJ, Love SB, Altman DG Survival analysis part II: multivariate data analysis--an introduction to concepts and methods. Br J Cancer. 2003 Ago;89(3):431-6.

11. Magrath I, Steliarova-Foucher $E$, Epelman S, Ribeiro RC, Harif M, Li C-K, Kebudi R, Macfarlan SD, Howard SC. Paediatric cancer in low-income and middle-income countries. Lancet Oncol. 2013 Mar;14(3):e104-16.

12. da Luz JF. Perfil Clínico-Demográfico dos Pacientes Atendidos no Serviço de Oncologia Pediátrica do Hospital de Clínicas de Porto Alegre: Período de Jan/2000 a Dez/2010 [dissertação]. Porto Alegre (RS): Universidade Federal do Rio Grande do Sul; 2011.

13. Doro MP, Pasquini R, Löhr SS. A functional assessment of adolescents who were recipients of bonemarrow transplantation: a prospective study. Rev. bras. hematol. hemoter. 2003; 25(1):5-15.

14. Strunk CJ, Alexander SW. Solid Tumors of Childhood. In: Chang AE, Hayes DF, Pass HI, Stone RM, Ganz PA, Kinsella TJ, Schiller JH, Strecher VJ, editores. Oncology: An Evidence-Based
Approach. New York: Springer; 2006. p. 1133-1156.

15. Li J, Thompson TD, Miller JW, Pollack LA, Stewart SL. Cancer incidence among children and adolescents in the United States, 2001-2003. Pediatrics. 2008 Jun 1;121:e1470-7.

16. Pritchard-Jones $K, \quad K a a t s c h \quad P$, Steliarova-Foucher E, Stiller CA, Coebergh JW. Cancer in children and adolescents in Europe: developments over 20 years and future challenges. Eur J Cancer. 2006 Set;42(13):2183-90.

17. Howlader $N$, Noone AM, Krapcho $M$, Neyman N, Aminou R, Waldron W. et al., editores. SEER Cancer Statistics Review, 1975-2009 (Vintage 2009 Populations) [Internet]. Bethesda (MD): National Cancer Institute; 2012 Apr [citado 2014 Mai 12]. Disponível em:

http://seer.cancer.gov/csr/1975 2009 _pops09/.

18. Magnani C, Pastore G, Coebergh JW, Viscomi S, Spix C, Steliarova-Foucher E. Trends in survival after childhood cancer in Europe, 1978-1997: report from the Automated Childhood Cancer Information System project (ACCIS). Eur J Cancer. 2006 Set;42(13):19812005.

19. Eyre TA, King AJ, Collins GP. Classical Hodgkin's lymphoma: past, present and future perspectives. $\mathrm{Br} J$ Hosp Med (Lond). 2013 Nov;74(11):612-8.

20. Neerav N, Shukla MD, Tanya $M$, Trippett MD. Non-Hodgkin's lymphoma in children and adolescents. Curr Oncol Rep. 2006;8(5):387-94.

21. Custódio $G$, Komechen $H$, Figueiredo FR, Fachin ND, Pianovski MA, Figueiredo BC. Molecular epidemiology of adrenocortical tumors in southern Brazil. Mol Cell Endocrinol. 2012 March; 351 (1):44-51.

22. Pereira Rosana Marques, Michalkiewicz Edson, Sandrini Fabiano, Figueiredo Bonald C., Pianovski Mara, 


\section{REVISTA MÉDICA DA UFPR}

França Susana Nesi et al. Tumores do córtex adrenal na infância. Arq Bras Endocrinol. 2004 Oct; 48( 5 ): 651-658.

23. Mirra AP, Latorre MRDO, Veneziano $D B$, editores. Incidência, mortalidade e sobrevida do câncer da infância no município de São Paulo. São Paulo (SP): Tomgraf Editora; 2004.

24. Rodriguez-Galindo $C$, Friedrich $P$, Morrissey L, Frazier L. Global challenges in pediatric oncology. Curr. Opin. Pediatr. 2013 Feb;25(1):3-15.

25. Armenian SH. Improving Screening Practices in Childhood Cancer Survivors at Risk for TreatmentRelated Heart Failure. J Clin Oncol. 2014 Nov;pii: JCO.2014.58.5562. [Epub ahead of print].

26. Meadows AT. Pediatric cancer survivorship: research and clinical care. J Clin Oncol. 2006 Nov;24(32):5160-5. 